\title{
FINITE GROUPS THAT NEED MORE GENERATORS THAN ANY PROPER QUOTIENT
}

\author{
FRANCESCA DALLA VOLTA and ANDREA LUCCHINI
}

(Received 29 January 1996; revised 5 March 1996)

Communicated by L. Kovács

\begin{abstract}
A structure theorem is proved for finite groups with the property that, for some integer $m$ with $m \geq 2$, every proper quotient group can be generated by $m$ elements but the group itself cannot.
\end{abstract}

1991 Mathematics subject classification (Amer. Math. Soc.): 20D20.

\section{Introduction}

Let $L$ be a non-cyclic finite group with a unique minimal normal subgroup, $M$. If $M$ is abelian, assume also that $M$ has a complement in $L$. Denote by $d(L)$ the minimum of the cardinalities of the generating sets of $L$.

For each positive integer $k$, let $L^{k}$ be the $k$-fold direct power of $L$ and define the subgroup $L_{k}$ by

$$
L_{k}=\left\{\left(l_{1}, \ldots, l_{k}\right) \in L^{k} \mid l_{1} \equiv \cdots \equiv l_{k} \bmod M\right\}
$$

Equivalently, set $\operatorname{diag} L^{k}=\left\{(l, \ldots, l) \in L^{k} \mid l \in L\right\}$ and $L_{k}=M^{k} \operatorname{diag} L^{k}$. It is easy to see that the socle of $L_{k}$ is $M^{k}$, a direct product of $k$ minimal normal subgroups (each isomorphic to $M$ ), and that $L_{k} / M^{k} \cong L / M$. The quotient group of $L_{k+1}$ over any minimal normal subgroup is isomorphic to $L_{k}$; in particular, the unbounded sequence $d\left(L_{1}\right), \ldots, d\left(L_{k}\right), \ldots$ is non-decreasing, and, by a theorem proved in [10], $d\left(L_{k+1}\right) \leq d\left(L_{k}\right)+1$. Thus if $m \geq d(L)$ then there is a (unique) $k$ such that $d\left(L_{k}\right)=m<d\left(L_{k+1}\right)$ : set $f(m)=k+1$. We shall comment below on how the function $f$ (which of course depends on $L$ ) may be calculated.

(C) 1998 Australian Mathematical Society $0263-6115 / 98 \$ A 2.00+0.00$ 
In attempting to prove that each finite group with a certain property can be generated by $m$ elements, one frequently considers groups $H$ such that every proper quotient group of $H$ can be generated by $m$ elements but $H$ itself cannot. The main result of this paper is that if $m \geq 2$ then each such $H$ is one of the $L_{f(m)}$ constructed above. By definition, $f(m)$ is never 1 , so it is part of the claim that $H$ must have more than one minimal normal subgroup. This part was established in [11]; it depends heavily on the classification of the finite simple groups. There is no need for more work of that kind here. (We do not consider here the analogous problem of non-cyclic groups all of whose proper quotient groups are cyclic.)

This paper was motivated by an attempt at understanding finite groups that are minimal with respect to having non-zero presentation rank (in the sense that the presentation rank of each proper quotient group is 0 ). It was proved by Gruenberg in [6] that such a group has no abelian minimal normal subgroup and is an $H$ for some $m \geq 2$. In view of this, the main theorem implies that each group which is minimal with respect to having non-zero presentation rank is the $L_{f(d(L))}$ built from an $L$ with non-abelian $M$, and of course each quotient group of that $L / M$ must have presentation rank 0 . It is also shown here that, conversely, if an $L$ has non-abelian $M$ and is such that all quotient groups of $L / M$ have presentation rank 0 , then the $L_{f(d(L))}$ formed from that $L$ has presentation rank 1 and all its proper quotient groups have presentation rank 0 .

The first example of a group with non-zero presentation rank was given in Cossey, Gruenberg and Kovács [2] as $A_{5}^{20}$ where $A_{5}$ is the alternating group of degree 5. In the present notation, $A_{5}^{20}=L_{f(2)}$ with $L=A_{5}$. The result of the previous paragraph and a careful examination of the functions $f$ associated to other choices of $L$ may make it possible to confirm the old feeling that $A_{5}^{20}$ must be the smallest group with positive presentation rank.

For any finite group $G$, let $\phi_{G}(m)$ denote the number of $m$-bases of $G$, that is, ordered $m$-tuplets $\left(x_{1}, \ldots, x_{m}\right)$ of elements of $G$ that generate $G$. This function was introduced by Philip Hall [8] with the name of Eulerian function.

If $L$ is one of the groups described above, let $\Gamma$ denote the group of those automorphisms of $L$ that act trivially on $L / M$. Further, if $M$ is abelian, set $q=\left|\operatorname{End}_{L / M} M\right|$. We prove that if $m \geq d(L)$ then

$$
f(m)=1+ \begin{cases}\phi_{L}(m) /|\Gamma| \phi_{L / M}(m) & \text { if } M^{\prime}=M, \\ \log _{q}\left(1+(q-1) \phi_{L}(m) /|\Gamma| \phi_{L / M}(m)\right) & \text { if } M^{\prime}=1 .\end{cases}
$$

When $M$ is abelian, this and results of Gaschütz [5] lead to

$$
f(m)=1+\log _{q}\left(|M|^{m-1} /\left|H^{1}(L / M, M)\right|\right),
$$

which could have also been derived from Theorem 6 of Gaschütz [3]. When $L$ is a finite non-abelian simple group, $L_{k}$ coincides with the $k$ th direct power $L^{k}$; the sequence 
$\left\{d\left(L^{k}\right)\right\}$ is called the growth sequence and has been studied in a series of papers by James Wiegold ([13-16], etcetera). In this case $\Gamma=\operatorname{Aut} L$ and $\phi_{L / M}(m)=1$, so $f(m)=\left(\phi_{L}(m) / \mid\right.$ Aut $\left.L \mid\right)+1$, a well-known result, proved many years ago by Philip Hall [8].

We are very grateful to Professor Kovács for many useful suggestions and remarks.

\section{Section 1}

In this section $m$ is an integer with $m \geq 2$ and we consider finite groups $H$ such that every proper quotient group of $H$ can be generated by $m$ elements but $H$ itself cannot. It will be useful to recall some known results.

THEOREM 1.1 (Gaschütz [4]). Let $N$ be a normal subgroup of a finite group $G$ and let $g_{1}, \ldots, g_{m} \in G$ be such that $G=\left\langle g_{1}, \ldots, g_{m}, N\right\rangle$. If $d(G) \leq m$, then there exist elements $u_{1}, \ldots, u_{m}$ of $N$ such that $G=\left\langle g_{1} u_{1}, \ldots, g_{m} u_{m}\right\rangle$. Moreover the cardinality of the set $\left\{\left(u_{1}, \ldots, u_{m}\right) \in N^{m} \mid G=\left\langle g_{1} u_{1}, \ldots, g_{m} u_{m}\right\rangle\right\}$ is independent of the choice of $g_{1}, \ldots, g_{m}$.

THEOREM 1.2 ([10]). If $G$ is a finite group and $N$ is a minimal normal subgroup of $G$, then $d(G) \leq \max \{2, d(G / N)+1\}$.

THEOREM 1.3. If a finite non-cyclic group $G$ contains a unique minimal normal subgroup $M$, then $d(G)=\max \{2, d(G / M)\}$.

When $M$ is abelian this was proved by Aschbacher and Guralnick [1, Corollary 1] using the fact that the first cohomology group with coefficients in a faithful simple module is always strictly smaller than the module itself. The case $M$ non-abelian was considered in [11] and depends heavily on the classification of finite simple groups.

Throughout the paper, $L$ will always denote a non-cyclic finite group which has only one minimal normal subgroup, $M$, and $M$ will be either non-abelian or complemented in $L$. For each positive integer $k$, let $L^{k}$ be the $k$-fold direct power of $L$ and define the subgroup $L_{k}$ by

$$
L_{k}=\left\{\left(l_{1}, \ldots, l_{k}\right) \in L^{k} \mid l_{1} \equiv \cdots \equiv l_{k} \bmod M\right\} .
$$

Equivalently, $L_{k}=M^{k} \operatorname{diag} L^{k}$. The socle of $L_{k}$ is $M^{k}$, a direct product of $k$ minimal normal subgroups (each isomorphic to $M$ ), and $L_{k} / M^{k} \cong L / M$. It is easy to see that the quotient group of $L_{k+1}$ over any minimal normal subgroup is isomorphic to $L_{k}$; in particular, the sequence $d\left(L_{1}\right), \ldots, d\left(L_{k}\right), \ldots$ is non-decreasing, and, by Theorem 1.2, $d\left(L_{k+1}\right) \leq d\left(L_{k}\right)+1$. Thus if $m \geq d(L)$ then there is a unique $k$ such 
that $d\left(L_{k}\right)=m<d\left(L_{k+1}\right)$ : set $f(L, m)=k+1$. When $L$ can be identified from the context, we write $f(L, m)$ simply as $f(m)$.

The groups $L_{k}$ play a key role in the study of groups that need more generators than any proper quotient. Indeed we have:

THEOREM 1.4. Let $m$ be an integer with $m \geq 2$ and $H$ a finite group such that $d(H / N) \leq m$ for every non-trivial normal subgroup $N$, but $d(H)>m$. Then there is a group $L$ which has a unique minimal normal subgroup $M$ and is such that $M$ is either non-abelian or complemented in $L$ and $H \cong L_{f(L, m)}$.

ProOF. By Theorem 1.3, $H$ contains at least two different minimal normal subgroups. Suppose that $N_{1}, \ldots, N_{r}, \ldots$ are the minimal normal subgroups of $H$. As $d\left(H / N_{1}\right) \leq m$ by hypothesis, there are $m$ elements $h_{1}, \ldots, h_{m}$ of $H$ such that $H=$ $\left\langle h_{1}, \ldots, h_{m}, N_{1}\right\rangle$. Now consider $N_{r}$ with $r \neq 1$. Of course, $H=\left\langle h_{1}, \ldots, h_{m}, N_{1} N_{r}\right\rangle$ and, as $H / N_{1} N_{r}$ is isomorphic to the quotient $\left(H / N_{r}\right) /\left(N_{1} N_{r} / N_{r}\right)$ of $H / N_{r}$ and $H / N_{r}$ is $m$-generated, by Theorem 1.1 there exist $m$ elements $x_{1}, \ldots, x_{m} \in N_{1}$ such that $\left\langle h_{1} x_{1}, \ldots, h_{m} x_{m}, N_{r}\right\rangle=H$.

Consider the subgroup $K_{r}=\left\langle h_{1} x_{1}, \ldots, h_{m} x_{m}\right\rangle$. We claim that $N_{1}$ and $N_{r}$ are both complements for $K_{r}$ in $H$. Obviously $H=K_{r} N_{1}=K_{r} N_{r}$; so we have just to prove that $K_{r} \cap N_{i}$ is trivial $(i=1, r)$. As $\left[N_{1}, N_{r}\right]=1$, the intersection $K_{r} \cap N_{1}$ is a normal subgroup of $H=K_{r} N_{r}$. Since this normal subgroup is contained in the minimal normal subgroup $N_{1}$ of $H$, if $K_{r} \cap N_{1} \neq 1$ then $N_{1} \leq K_{r}$ and $H=K_{r} N_{1}=K_{r}$ is $m$-generated, which contradicts our hypothesis. The claim $K_{r} \cap N_{r}=1$ is proved similarly.

It is now easy to prove that the projections $\pi_{r}: K_{r} \cap\left(N_{1} \times N_{r}\right) \rightarrow N_{1}$ and $\rho_{r}: K_{r} \cap\left(N_{1} \times N_{r}\right) \rightarrow N_{r}$ are isomorphisms. Consider $\pi_{r}$ first: $\operatorname{ker} \pi_{r} \leq N_{r} \cap K_{r}=1$, so $\pi$, is injective. Moreover, for any $n_{1} \in N_{1}$ there exist $t \in K_{r}$ and $n_{r} \in N_{r}$ such that $n_{1}=t n_{r}:$ then $t=n_{1} n_{r}^{-1} \in\left(N_{1} \times N_{r}\right) \cap K_{r}$ and $t^{\pi_{r}}=n_{1}$, so $\pi_{r}$ is also surjective. Similar arguments can be applied to $\rho_{r}$.

What we shall use from this is that to each $r>1$ there is a subgroup $K_{r}$ which complements both $N_{1}$ and $N_{r}$ and an isomorphism $\phi_{r}: N_{1} \rightarrow N_{r}$ (namely $\phi_{r}=\pi_{r}^{-1} \rho_{r}$ ) such that $K_{r} \cap\left(N_{1} \times N_{r}\right)=\left\{x x^{\phi_{r}} \mid x \in N_{1}\right\}$. Using that this intersection is normal in $K_{r}$, it is easy to see that $\phi_{r}$ is a $K_{r}$-isomorphism.

When $N_{1}$ is abelian, the $\phi_{r}$ are in fact $H$-isomorphisms. We have proved that in this case each minimal normal subgroup of $H$ is abelian and complemented, so the Frattini subgroup of $H$ is trivial, and this implies that soc $H$ admits a complement, $K$ say. We conclude that $H \cong L_{k}$ where $L$ is the semidirect product $N_{1} K$ and $k$ is a suitable integer.

Now assume that $N_{1}$ is non-abelian. For this case, we choose $k$ so that the minimal normal subgroups of $H$ are $N_{1}, \ldots, N_{k}$. Let $\alpha_{1}: H \rightarrow$ Aut $N_{1}$ be the homomorphism 
defined by the conjugation action of $H$ on $N_{1}$, so

$$
h^{\alpha_{1}}: x \mapsto x^{h} \quad \text { whenever } h \in H, x \in N_{1},
$$

and let $L$ denote the image of $\alpha_{1}$. This group has only one minimal normal subgroup, namely the non-abelian group $M=\operatorname{Inn} N_{1}$. For $r>1$, define $\alpha_{r}: H \rightarrow$ Aut $N_{1}$ by

$$
h^{\alpha_{r}}: x \mapsto\left(\left(x^{\phi_{r}}\right)^{h}\right)^{\phi_{r}^{-1}} \quad \text { whenever } \quad h \in H, x \in N_{1} .
$$

As $H=K_{r} N_{1}$, we can write each $h$ as $h=u v$ with $u \in K_{r}, v \in N_{l}$, and then

$$
\left(\left(x^{\phi_{r}}\right)^{h}\right)^{\phi_{r}^{-1}}=\left(\left(x^{\phi_{r}}\right)^{u}\right)^{\phi_{r}^{-1}}=x^{u}=\left(x^{h}\right)^{v^{-1}}
$$

where the first equality holds because $N_{1}$ centralizes $N_{r}$ and the second because $\phi_{r}$ is a $K_{r}$-map: thus $h^{\alpha}$ is $h^{\alpha_{1}}$ followed by the inner automorphism of $N_{1}$ induced by $v^{-1}$. We conclude that

$$
h^{\alpha_{1}} \equiv \cdots \equiv h^{\alpha_{k}} \bmod M \text {. }
$$

In particular, it follows that each $\alpha_{r}$ has image $L$, and the $\alpha_{r}$ together yield a homomorphism from $H$ onto $L_{k}$. The kernel of this homomorphism is the intersection of the kernels of the $\boldsymbol{\alpha}_{r}$, that is, of the centralizers of the $N_{r}$ (with $r=1, \ldots, k$ ): that intersection being trivial, our homomorphism $H \rightarrow L_{k}$ is an isomorphism.

Regardless of whether $N_{1}$ is abelian, we have proved that $H \cong L_{k}$ with $k \geq 2$, whence it follows that $H / N_{1} \cong L_{k-1}$. Thus $d\left(L_{k-1}\right) \leq m<d\left(L_{k}\right)$. By the definition of the function $f$, this means that $k=f(L, m)$, and the proof of the theorem is complete.

\section{Section 2}

The aim of this section is to describe how the function $f$ can be evaluated.

LEMMA 2.5. Given a homomorphism from an $L_{k}$ onto $L / M$, consider the set $\mathscr{S}$ of normal subgroups $N$ of $L_{k}$ arising as kernels of those homomorphisms of $L_{k}$ onto $L$ which composed with the natural $L \rightarrow L / M$ yield the given $L_{k} \rightarrow L / M$. The cardinality of the set $\mathscr{S}$ is $k$ when $M$ is non-abelian; it is $\left(q^{k}-1\right) /(q-1)$ when $M$ is abelian and $q$ is the number of $(L / M)$-endomorphisms of $M$.

PROOF. If $\beta: L_{k} \rightarrow L / M$ is a surjective homomorphism, then $\operatorname{ker} \beta=\operatorname{soc} L_{k}=$ $M^{k}$. So the normal subgroups $N$ we have to count are precisely the normal subgroups of $L_{k}$ contained in $\operatorname{soc} L$ and such that $L_{k} / N \cong L$. If $M$ is non-abelian then the $k$ 
direct factors of $M^{k}$ are the unique minimal normal subgroups of $L_{k}$ and the normal subgroups $N$ we are considering are precisely the direct products of $k-1$ of them, so we have exactly $k$ possibilities. If $M$ is abelian we have to count the kernels of surjective $(L / M)$-homomorphisms from $M^{k}$ to $M$ and there are $\left(q^{k}-1\right) /(q-1)$ of these where $q$ is the number of $(L / M)$-endomorphisms of $M$.

For any finite group $G$, let $\phi_{G}(m)$ denote the number of $m$-bases of $G$, that is, ordered $m$-tuplets $\left(x_{1}, \ldots, x_{m}\right)$ of elements of $G$ that generate $G$. This function was introduced by Philip Hall [8] with the name of Eulerian function.

In [5] Gaschütz studied Eulerian functions for solvable groups. Here we generalize some of the ideas contained in [5] to the non-solvable case.

Let $\Gamma$ denote the group of those automorphisms of $L$ that act trivially on $L / M$.

LEMMA 2.6. Let $F$ be a free group of rank $m \geq d(L)$. Given a homomorphism from $F$ onto $L / M$, consider the set $\mathscr{R}$ of normal subgroups $N$ of $F$ arising as kernels of those homomorphisms of $F$ onto $L$ which composed with the natural $L \rightarrow L / M$ yield the given $F \rightarrow L / M$. The cardinality of the set $\mathscr{R}$ is $\phi_{L}(m) /|\Gamma| \phi_{L / M}(m)$.

PROOF. Let $x_{1}, \ldots, x_{m}$ be a basis of $F$. A surjective homomorphism $\beta: F \rightarrow$ $L / M$ is uniquely determined by $\beta\left(x_{1}\right)=l_{1} M, \ldots, \beta\left(x_{m}\right)=l_{m} M$, where $L=$ $\left\langle l_{1}, \ldots, l_{m}, M\right\rangle$. Now let $\gamma: F \rightarrow L$ be a surjective homomorphism which composed with the natural $L \rightarrow L / M$ yields $\beta$; we must have $\gamma\left(x_{1}\right)=l_{1} z_{1}, \ldots, \gamma\left(x_{m}\right)=l_{m} z_{m}$ with $z_{1}, \ldots, z_{m} \in M$ and $L=\left\langle l_{1} z_{1}, \ldots, l_{m} z_{m}\right\rangle$. By Theorem 1.1 the number of possible choices for $\left(z_{1}, \ldots, z_{m}\right)$ is $\phi_{L}(m) / \phi_{L / M}(m)$, independently of the choice of $\left(l_{1}, \ldots, l_{m}\right)$; so the number of possibilities for $\gamma$ is $\phi_{L}(m) / \phi_{L / M}(m)$. Now let $\gamma_{1}, \gamma_{2}$ be two of these homomorphisms; $\operatorname{ker} \gamma_{1}=\operatorname{ker} \gamma_{2}=N$ if and only if there exists an automorphism $\alpha$ of $L$ which acts trivially on $L / M$ such that $\gamma_{2}$ is equal to $\gamma_{1}$ composed with $\alpha$. We conclude that the cardinality of $\mathscr{R}$ is $\phi_{L}(m) /|\Gamma| \phi_{L / M}(m)$.

We can now prove the main result of this section:

THEOREM 2.7. If $m \geq d(L)$ then, with $q=\left|\operatorname{End}_{L / M} M\right|$,

$$
f(m)=1+ \begin{cases}\phi_{L}(m) /|\Gamma| \phi_{L / M}(m) & \text { if } M^{\prime}=M, \\ \log _{q}\left(1+(q-1) \phi_{L}(m) /|\Gamma| \phi_{L / M}(m)\right) & \text { if } M^{\prime}=1 .\end{cases}
$$

PROOF. Let $F$ be a free group of rank $m$. Given a surjective homomorphism $\beta: F \rightarrow L$, consider the set $\mathscr{R}$ defined in Lemma 2.6 and let $R=\bigcap_{N \in \mathscr{R}} N$. It is easy to see that $F / R \cong L_{k}$ for some $k$ depending on the $\operatorname{rank} m$ of $F$, and that $F / R$ is the largest quotient of $F$ isomorphic to $L_{i}$ for some $i$; this means that $f(m)=1+k$. Now $\beta$ induces a surjective homomorphism $\bar{\beta}: F / R \cong L_{k} \rightarrow L / M$ and the map 
$N \mapsto N / R$ is a bijection between $\mathscr{R}$ and the set $\mathscr{S}$ defined in Lemma 2.5. So we have, by Lemma 2.5 and Lemma 2.6 ,

$$
\frac{\phi_{L}(m)}{|\Gamma| \phi_{L / M}(m)}=|\mathscr{R}|=|\mathscr{S}|= \begin{cases}k & \text { if } M^{\prime}=M, \\ \left(q^{k}-1\right) /(q-1) & \text { if } M^{\prime}=1 .\end{cases}
$$

Since $k=f(m)-1$, this completes the proof.

For the case of abelian $M$, Gaschütz proved ([5, Theorem 2]) that

$$
\phi_{L}(m) / \phi_{L / M}(m)=|M|^{m}-a
$$

where $a$ is the number of complements of $M$ in $L$. Since $a=|M|\left|H^{1}(L / M, M)\right|$ while $|\Gamma|=(q-1) a$, one concludes that

$$
f(m)=1+\log _{q}\left(|M|^{m-1} /\left|H^{1}(L / M, M)\right|\right) .
$$

This form of our result could be deduced more directly from Theorem 6 of Gaschütz [3].

When $L$ is a non-abelian simple group, what Theorem 2.7 gives is that

$$
f(m)=\left(\phi_{L}(m) / \mid \text { Aut } L \mid\right)+1,
$$

a well-known result, proved many years ago by Philip Hall [8]. In any case, Aut $L$ permutes the $m$-bases regularly and $\phi_{L}(m) / \mid$ Aut $L \mid$ is the number of orbits. A similar interpretation can be given for the number $\phi_{L}(m) / \phi_{L / M}(m)|\Gamma|$. Fix $\left(l_{1}, \ldots, l_{m}\right) \in L^{m}$ with the property that $\left\langle l_{1}, \ldots, l_{m}, M\right\rangle=L$ and consider the set

$$
\Omega=\left\{\left(\bar{l}_{1}, \ldots, \bar{l}_{m}\right) \in L^{m} \mid L=\left\langle\bar{l}_{1}, \ldots, \bar{l}_{m}\right\rangle, l_{i} \equiv \bar{l}_{i} \bmod M \text { for each } 1 \leq i \leq m\right\} .
$$

The subgroup $\Gamma$ of Aut $L$ stabilizes $\Omega$ and $\phi_{L}(m) / \phi_{L / M}(m)|\Gamma|$ is the number of orbits for the action of $\Gamma$ on $\Omega$ (and is independent, by Theorem 1.1, of the choice of $\left.l_{1}, \ldots, l_{m}\right)$.

\section{Section 3}

The presentation $\operatorname{rank} \operatorname{pr}(G)$ of a finite group $G$ is an invariant whose definition comes from the study of relation modules (see [7] for more details). It also plays a role in the study of the minimal number of generators $d(G)$ of $G$. Let $I_{G}$ denote the augmentation ideal of $\mathbb{Z} G$, and $d\left(I_{G}\right)$ the minimal number of elements of $I_{G}$ needed to generate $I_{G}$ as a $G$-module. Roggenkamp [12] proved that $d(G)=d\left(I_{G}\right)+\operatorname{pr}(G)$. It is 
known that $\operatorname{pr}(G)=0$ for many groups $G$, including all solvable groups, all Frobenius groups and all 2-generator groups, but examples of groups with presentation rank $n$ can be constructed for every positive integer $n$. On the other hand, the only known examples of groups with non-zero presentation rank are sufficiently high direct powers of perfect groups and groups related to them. The smallest example which is known is the direct product $A_{5}^{20}$ of 20 copies of the alternating group of degree 5. It is an open problem whether it is possible to construct examples of different kinds or whether there exists a group $G$ with $\operatorname{pr}(G) \neq 0$ and $|G|<\left|A_{5}^{20}\right|$.

In this section we study groups that are minimal with respect to having non-zero presentation rank (in the sense that the presentation rank of each proper quotient group is 0 ).

Gruenberg proved $([6,(2.4)]$ and $[7$, Proposition 6.2]):

THEOREM 3.8. If $H$ is minimal with respect to having non-zero presentation rank, then $H$ contains no non-trivial solvable normal subgroups, $d(H)>2$, and $d(H / N)<$ $d(H)$ for every non-trivial normal subgroup $N$ of $H$.

To continue the study of the structure of these groups the following result is useful. It can be considered as a particular case of a theorem proved by Kimmerle and Williams [9, Theorem 4.3], but it is also implicit in earlier papers, for example in [2] and in [6].

LEMMA 3.9. If $H$ contains a non-trivial normal subgroup $N$ all of whose chief factors are non-abelian, then $d\left(I_{H}\right)=\max \left\{2, d\left(I_{H / N}\right)\right\}$.

We can now prove

THEOREM 3.10. Each group which is minimal with respect to having non-zero presentation rank is the $L_{f(L . d(L))}$ built from an $L$ with non-abelian $M$, and of course each quotient group of that $L / M$ must have presentation rank 0 . Conversely, if an $L$ has non-abelian $M$ and is such that all quotient groups of $L / M$ have presentation rank 0 , then the $L_{f(d(L))}$ formed from that $L$ has presentation rank 1 and all its proper quotient groups have presentation rank 0.

PROOF. By Theorem 3.8 and Theorem 1.4, a group which is minimal with respect to having non-zero presentation rank is the $L_{f(m)}$ built from an $L$ with non-abelian $M$. Of course each quotient group of $L / M$, being a proper quotient group of $L_{f(m)}$, must have presentation rank 0 . As $L_{f(m)} / M^{f(m)} \cong L / M$, we have that

$$
d\left(I_{L_{f(m i)}}\right)=\max \left\{2, d\left(I_{L / M}\right)\right\}=\max \{2, d(L / M)\}=d(L)
$$

(by Lemma 3.9 and Theorem 1.3), while $d\left(L_{f(m)}\right)=m+1$ (by Theorem 1.2), so $\operatorname{pr}\left(L_{f(m)}\right)=m+1-d(L) \geq 1$, with equality if and only if $m=d(L)$. Since $L_{f(d(L))}$ is a quotient group of $L_{f(m)}$ whenever $m \geq d(L)$, the first claim now follows. 
Conversely, suppose that $M$ is non-abelian and that all quotient groups of $L / M$ have presentation rank 0 . We noted above that $\operatorname{pr}\left(L_{f(d(L))}\right)=1$. A proper quotient group of $L_{f(d(L))}$ is isomorphic either to a quotient group of $L / M$ or to an $L_{k}$ with $k<f(d(L))$. In the former case it has presentation rank 0 by hypothesis, while in the latter case it has presentation rank 0 because

$$
\operatorname{pr}\left(L_{k}\right)=d\left(L_{k}\right)-d\left(I_{L_{k}}\right)=d(L)-\max \{2, d(L / M)\}=0 .
$$

This completes the proof of the theorem.

To conclude we note that, analyzing in more detail the proof of Theorem 1.3, one notices that the number $\phi_{L}(m) / \phi_{L / M}(m)$ is in general quite large (for example $\left.\phi_{L}(m) / \phi_{L / M}(m) \geq\left|M^{m-2}\right|\right)$, so it should be possible to prove that $f(m)$ is also large. So we have the following informal interpretation of Theorem 3.10: a finite group with non-zero presentation rank should contain a section isomorphic to the direct product of 'many' copies of a non-abelian simple group.

\section{References}

[1] M. Aschbacher and R. Guralnick, 'Some applications of the first cohomology group', J. Algebra 90 (1984), 446-460.

[2] J. Cossey, K. W. Gruenberg and L. G. Kovács, 'The presentation rank of a direct product of finite groups', J. Algebra 28 (1974), 597-603.

[3] W. Gaschütz, 'Über modulare Darstellungen endlicher Gruppen, die von freien Gruppen induziert werden', Math. Z. 60 (1954), 274-286.

[4] — ' ' Zu einem von B. H. und H. Neumann gestellten Problem', Math. Nachr. 14 (1955), 249-252.

[5] - 'Die eulersche funktion endlicher auflösbarer gruppen', Illinois J. Math. 3 (1959), 469-476.

[6] K. W. Gruenberg, 'Groups of non-zero presentation rank', Symposia Math. 17 (1976), 215-224.

[7] _ - Relation modules of finite groups, CBMS No. 25 (Amer. Math. Soc., Providence, 1976).

[8] P. Hall, 'The Eulerian functions of a group', Quart. J. Math. 7 (1936), 134-151.

[9] W. Kimmerle and J. S. Williams, 'On minimal relation modules and 1-cohomology of finite groups', Arch. Math. 42 (1984), 214-223.

[10] A. Lucchini, 'Generators and minimal normal subgroups', Arch. Math. 64 (1995), 273-276.

[11] A. Lucchini and F. Menegazzo, 'Generators for finite groups with a unique minimal normal subgroup', Rend. Sem. Mat. Univ. Padova, to appear.

[12] W. Roggenkamp, 'Relation modules of finite groups and related topics', Algebra i Logika 12 (1973), 351-369.

[13] J. Wiegold, 'Growth sequences of finite groups', J. Austral. Math. Soc. 17 (1974), 133-141.

[14] — - 'Growth sequences of finite groups II', J. Austral. Math. Soc. 20 (1975), 225-229.

[15] — _ 'Growth sequences of finite groups III', J. Austral. Math. Soc. Ser. A 25 (1978), 142-144.

[16] ___ 'Growth sequences of finite groups IV', J. Austral. Math. Soc. Ser. A 29 (1980), 14-16. 
Dipartimento di Matematica "F. Enriques" Università di Milano

Via Saldini 50, 20133 Milano

Italy

e-mail: dallavolta@vmimat.mat.unimi.it
Dipartimento di Elettronica per l'Automazione Università di Brescia Via Branze 38, 25123 Brescia

Italy e-mail: lucchini@bsing.ing.unibs.it 